\title{
Recomendar contenidos digitales para niños y jóvenes: reflexiones, herramientas y criterios
}

\author{
Luis Miguel Cencerrado Malmierca \\ Universidad de Valladolid, España \\ luismiguel.cencerrado@uva.es

\section{Sofia Pelosi} \\ Universidad de Salamanca, España \\ sofia.pelosi01@usal.es \\ Elisa Yuste Tuero \\ Consultoría en Cultura y Lectura, España \\ info@elisayuste.com
}

Cita sugerida: Cencerrado Malmierca, L. M.; Pelosi, S. y Yuste Tuero , E. (2018). Recomendar contenidos digitales para niños y jóvenes: reflexiones, herramientas y criterios. Palabra Clave (La Plata), 7(2), e046. https://doi.org/10.24215/18539912e046 


\section{Recomendar contenidos digitales para niños y jóvenes: reflexiones, herramientas y criterios}

Reviewing digital contents for children and teens: reflections, tools and criteria

Luis Miguel Cencerrado Malmierca

Universidad de Valladolid, España

luismiguel.cencerrado@uva.es

Sofia Pelosi

Universidad de Salamanca, España

sofia.pelosi01@usal.es

Elisa Yuste Tuero

Consultoría en Cultura y Lectura, España

info@elisayuste.com

\section{Resumen:}

El artículo plantea la importancia y necesidad de las plataformas de recomendación de aplicaciones y otros materiales de lectura digitales de ficción y no ficción dirigidas a niños y jóvenes. Primero se justifica el papel de los recomendadores como herramientas facilitadoras de la labor de padres, docentes, bibliotecarios y otros agentes promotores de la lectura digital. Se analiza después la caracterización de las plataformas existentes a partir de la evaluación de las consideradas más significativas. Finalmente se ponderan los aspectos fundamentales que deben abordar los recomendadores, e igualmente se hace un repaso a los parámetros y criterios de evaluación para determinar la calidad de las aplicaciones para niños y jóvenes.

Palabras clave: App para niños y jóvenes, Lectura digital, Promoción de la lectura, Recomendadores de lecturas, Evaluación de materiales de lectura.

\section{Abstract:}

The article is focusing on the importance and necessity of reviewing apps and digital media contents for children and teens, particularly fiction and no-fiction contents. The first part is centred on the main role of rating and reviews as an essential tool for parents, teachers and librarians by providing unbiased information and trusted advice. The next one analyses the characteristics of selected blogs and websites that offer reviews and rating. The last part consider which aspects are fundamental in order to define "a good quality" app for children and teens.

KEYWORDS: App for children and teens, Digital reading, Promoting reading skills, Reading recommendations, Rate reading contents.

\section{RECOMENDADORES DE APPS INFANTILES Y JUVENILES: ¿NECESARIOS Y PARA QUÉ?}

Las apps y otros recursos digitales son una nueva puerta de acceso a la experiencia literaria y al conocimiento. La oferta de este tipo de materiales de lectura no para de crecer, y dentro de ella las apps destinadas a niños y jóvenes tienen un significativo peso específico. Estos materiales digitales invitan a niños y jóvenes a explorar nuevas formas de contar historias, a aprehender la realidad de manera diferente, brindándoles también oportunidades de expresarse, de crear y de comunicarse con otros lectores.

Como se recoge en un reciente informe del Observatorio de la Lectura y el Libro (2017, p. 76), la lectura digital aumenta su presencia de forma constante en el subsector de la edición para niños y jóvenes:

El libro infantil juvenil, al igual que el resto del sector del libro, está en plena transformación provocada por la revolución tecnológica en la que estamos inmersos. El libro digital está aquí para quedarse y convivir con el impreso. Aunque en el mercado mundial del libro en general, y en el español en particular, todavía ofrece cifras modestas y parece que no acaba de despegar, al menos no de forma tan radical como algunos predijeron, incluso hay quien dice que se ha estancado, es una 
realidad que está afectando a nuestra vida y que seguirá transformando los hábitos lectores de todos, también los de los niños y jóvenes.

En efecto, el conjunto de la oferta de ficción y de no ficción en formato digital ha experimentado un significativo desarrollo en los últimos años y conforma un panorama cada vez más diverso y atractivo. Por otro lado, en la medida en que este sector crece resulta más complicado identificar estos materiales, así como determinar el carácter y la calidad de los mismos. Conocer qué tipos de obras componen la oferta, así como hacerse una idea de su valor, del interés de la propuesta de lectura que ofrecen y de la pertinencia de sus contenidos, no resulta una labor fácil para muchos mediadores y promotores de la lectura que se acercan al mundo digital. Incluso puede suponerles no pocos quebraderos de cabeza al no contar con las herramientas necesarias para conocer lo que se publica y guiar la mirada de los padres, madres, docentes o bibliotecarios interesados en promover la lectura digital en sus diferentes ámbitos de actuación.

De ahí que se precisen estos espacios y herramientas de información y orientación, para dar a conocer la oferta de apps destinadas al público infantil y juvenil, por un lado, y orientar la labor de selección en la familia, la escuela y la biblioteca, por otro. Las aplicaciones dirigidas al público infantil y juvenil conforman un panorama cada vez más diverso y atractivo que requiere de mayor atención para destacar aquellas que encierran un especial interés, poniendo de manifiesto:

- La riqueza y diversidad que aporta la interrelación de los diferentes códigos que las componen: textual y gráfico, ilustraciones, animaciones, vídeos, sonido y música.

- La amplitud de funciones a las que responden: para leer, escuchar, ver, escribir, aprender, crear, jugar, comunicarse o compartir y disfrutar en compañía.

- La exploración de nuevos caminos narrativos y la incorporación de recursos estéticos renovadores que emanan del diálogo entre los múltiples elementos que componen las obras digitales, de su carácter híbrido e integrador de diferentes lenguajes.

- Las diferentes formas de presentar la información que ofrecen, en un intento de renovar el modo de acceder al conocimiento y de propiciar aprendizajes personalizados, activos y significativos para el lector.

- Las experiencias híbridas de lectura que brindan a los lectores, con buenas dosis de interacción y propuestas enriquecidas, tales como la Realidad Aumentada o la Realidad Virtual que favorecen una mayor inmersión en las historias e intensifican la relación del lector con todo tipo de contenidos.

La figura 1 recoge la infografía elaborada por una de las plataformas de recomendación existentes, AppTK.es, en la que se muestran de una manera sintética los caminos por los que discurren hoy las apps para niños y jóvenes y se apuntan las tendencias de evolución de estos materiales de lectura. 


\section{0 tendencias en apps para niños y jóvenes}

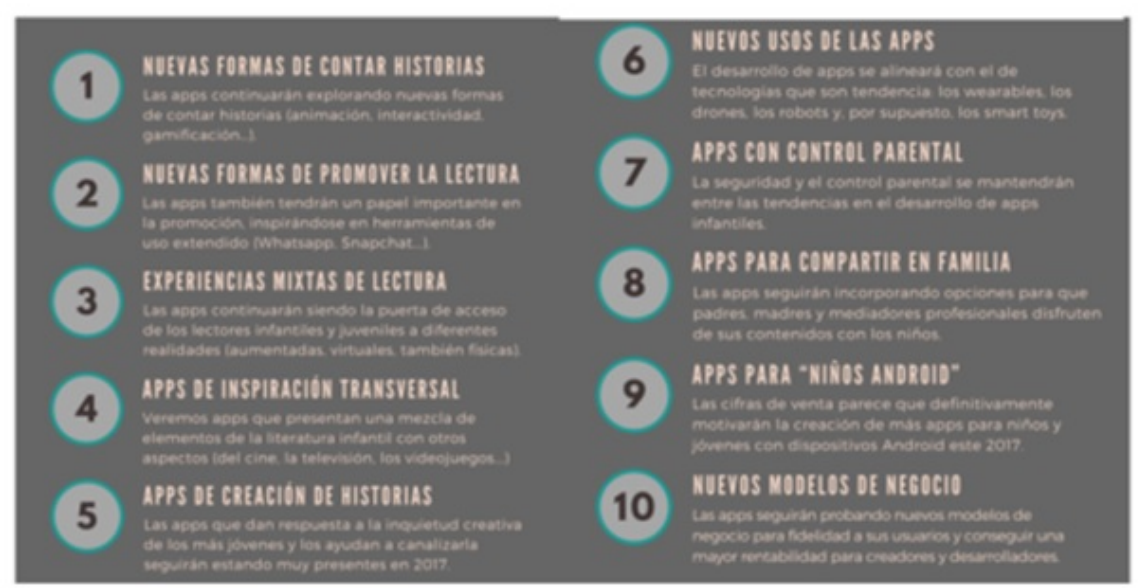

FIGURA 1

Tendencias en apps para niños y jóvenes

AppTK.es - http://apptk.es/infografia-10-tendencias-en-apps-para-ninos-y-jovenes-2017/

\section{EL Reto DE HACER VISIBLE LA OFERTA}

La oferta de materiales de lectura para niños y jóvenes, ya de por sí muy extensa y difícilmente abarcable en el formato impreso, se incrementa con la aparición de las obras digitales, lo que supone un aumento de las dificultades para conocer qué es lo que se publica y qué valor tienen estas obras. Dar visibilidad a las apps y difundir el conjunto de la oferta digital es una necesidad urgente para que la lectura en pantalla se desarrolle con normalidad, para que se conozca qué se está haciendo en este terreno y para facilitar que estas obras entren a formar parte del día a día de los lectores en los hogares, las escuelas y las bibliotecas. La creación de espacios de orientación y recomendación crítica es la mejor vía para hacer frente a la sensación de saturación que provoca en los mediadores el extenso panorama de la oferta, la diversidad de tipologías de los materiales de lectura a la que se tienen que enfrentar y el amplio abanico de calidades que hay detrás de cada una de estas obras.

Ciertamente, esta eclosión de las aplicaciones para niños provoca cierto vértigo y desconcierto a los mediadores que se acercan en cierta manera desarmados y que pretenden seleccionar materiales adecuados para sus hijos, sus alumnos o usuarios en la familia, la escuela, la biblioteca o de otros ámbitos. Para salvar estas dificultades es necesario, por tanto, poner a su alcance herramientas que permitan explorar con confianza este sector de la edición digital y que aporten fiabilidad a los destinatarios de este tipo de literatura (niños, padres, maestros y bibliotecarios), así como a los propios editores y desarrolladores. Los buscadores y plataformas de análisis y recomendación de apps se dibujan como un apoyo esencial al servicio de todos los implicados en la creación, desarrollo, distribución y promoción de la lectura digital. Suponen igualmente un valioso recurso para que los mediadores conozcan los desarrollos actuales, estén al día de las novedades y para facilitar su labor de promoción de la lectura, tanto en papel como en pantalla. 


\section{LA NECESIDAD DE INTERMEDIACIÓN, TAMBIÉN EN EL ENTORNO DIGITAL}

La presencia de tabletas y teléfonos inteligentes en los hogares sigue creciendo y el contacto de los más jóvenes con este tipo de dispositivos móviles se adelanta a edades más tempranas. Según informes recientes como el We are social (2017), el 92\% de los españoles son usuarios de algún tipo de teléfono móvil y el $81 \%$ dispone de un smartphone con conexión a internet. Lógicamente, a la par que el parque tecnológico crece también lo hacen las aplicaciones instaladas en todos estos dispositivos, cuya cantidad y diversidad de funcionalidades aumenta de forma continua.

A pesar de ello, entre los usos mayoritarios que los españoles hacen de tabletas, teléfonos inteligentes y otros dispositivos móviles, la lectura no aparece más que de una forma residual, según nos recuerdan de forma recurrente los informes que monitorizan el sector. Esta ausencia es una llamada de atención sobre la necesidad de desarrollar planes de formación en el uso de los dispositivos electrónicos, de cara a ampliar las posibilidades que estos ofrecen y para dar sentido a "los cacharros" en relación con la lectura y la escritura. Es urgente, en consecuencia, plantearse seriamente políticas y estrategias de actuación al respecto, a través de la mediación de los agentes formativos, culturales y sociales, en los espacios tanto de educación como no formal.

Las plataformas de recomendación de materiales de lectura digital pueden ser grandes aliadas para el desarrollo de esta labor divulgativa y formadora que se lleva a cabo en las distintas instituciones y organizaciones promotoras de la lectura. Es el caso de las escuelas y las bibliotecas, entre cuyas funciones primordiales está la recomendación y la orientación en materia de lectura. Los mediadores que se mueven en estos ámbitos, y en otros ámbitos de actuación, necesitan herramientas que les ayuden a conocer, evaluar y seleccionar las obras electrónicas para que puedan ejercer de una forma más ágil y eficaz esta labor de mediación y prescripción de lecturas contando también con la oferta digital.

\section{Seleccionar apps de lectura para niños y Jóvenes: S.O.S. ¿QuiÉn Me ayuda?}

Necesitamos pues, herramientas que identifiquen, analicen y evalúen lo que se produce en el espacio digital, y que respalden la tarea de recomendación de padres, docentes y bibliotecarios, así como de otros agentes como los libreros, etc. Prestar esta labor de asesoramiento y recomendación de lecturas digitales implica también aprovechar los recursos que el propio entorno digital proporciona, pues como apunta Maribel Riaza (2016) en la presentación de la infografía acerca de cómo encuentran los lectores su próxima lectura, las recomendaciones que circulan por el espacio digital son ya un complemento de las tradicionales prescripciones de libreros y bibliotecarios y las reseñas de los suplementos culturales de medios impresos. De esta manera las redes sociales, blogs, aplicaciones y las comunidades de lectores virtuales se configuran como nuevos e influyentes espacios de orientación y recomendación de lecturas.

También García-Rodríguez, Gómez-Díaz y Cordón-García (2013, p. 23), expertos en lectura digital, insisten en esta necesidad de ampliar las fuentes de información para que todos los interesados puedan manejarse con mayor soltura entre la oferta de materiales digitales de lectura:

Los consumidores de este tipo de literatura (niños, jóvenes, padres, maestros, bibliotecarios...), demandan, cada vez más, puntos de referencia que les permitan encontrar y seleccionar aquellos títulos más interesantes. Los profesionales de la LIJ se están viendo en la necesidad de desarrollar instrumentos de orientación bibliográfica que puedan satisfacer esta demanda, pero se encuentran con la falta de criterios específicos para la edición digital y la carencia y/o desconocimiento de recursos y fuentes que puedan utilizar en esta tarea.

Por suerte, el panorama de este tipo de recursos está creciendo en nuestro entorno, tanto en cantidad como en la calidad de su trabajo de evaluación y selección de lecturas digitales de ficción y de no ficción para niños y jóvenes (Figura 2). 


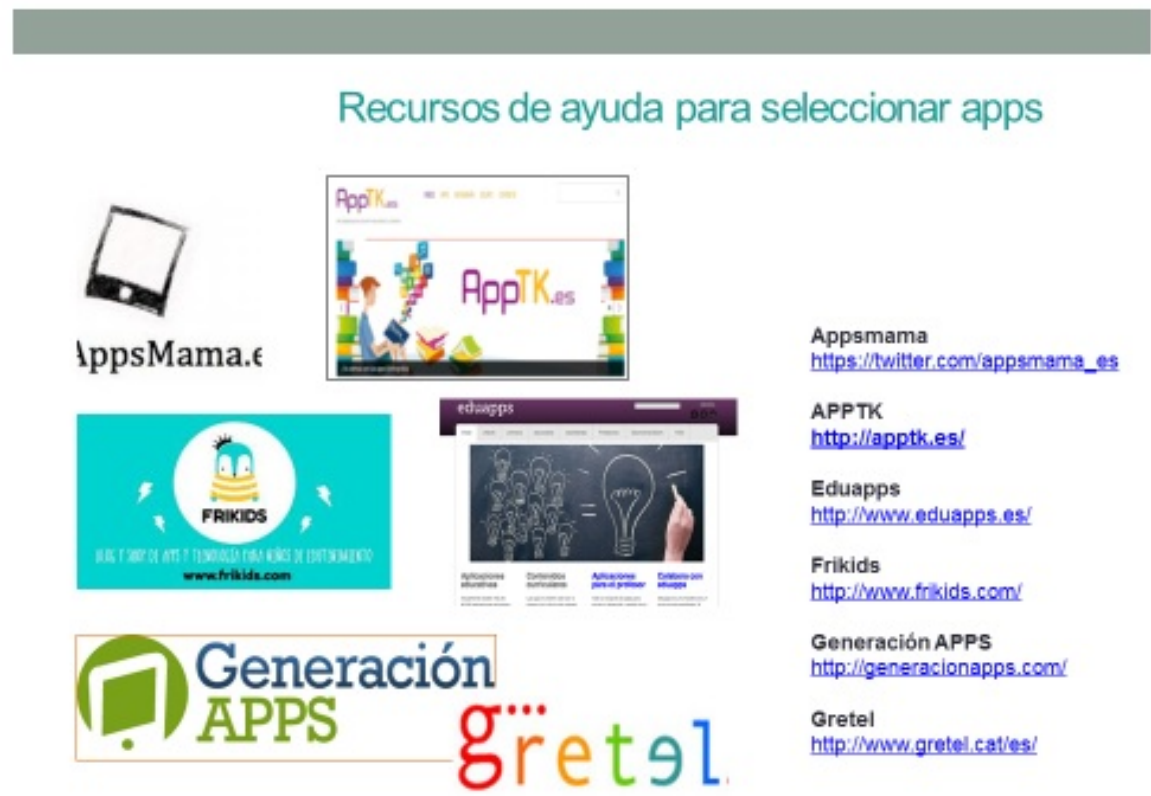

FIGURA 2

Espacios de recomendación de apps para niños y jóvenes Elaboración propia.

\section{5. ¿QUÉ LE PEDIMOS A UN BUEN RECOMENDADOR?}

Para que una plataforma de recomendación pueda llegar a tener un impacto real y constante en los destinatarios a los que pretende ayudar, no basta con reseñar de vez en cuando una serie de materiales y formular unos juicios de valor en función de no se sabe qué criterios y en razón de intenciones que no se explicitan.

Por el contrario, los espacios de orientación y recomendación que pretendan obtener la confianza de los mediadores deberían:

- Ser transparentes en su quehacer, es decir, definir de manera clara y explícita el propósito y los objetivos que alientan su trabajo de selección y recomendación.

- Configurarse como herramientas accesibles, completas y actualizadas que allanen el camino a quien las consulte a la hora de identificar, conocer y localizar las apps dirigidas a niños y jóvenes disponibles en las diferentes plataformas.

- Presentar de forma abierta los criterios que se utilizan para valorar la calidad de los elementos que componen las obras analizadas y la pertinencia de sus contenidos.

- Aportar una mirada crítica que vaya más allá de la presentación meramente descriptiva; es decir, mostrar los puntos fuertes y débiles de la oferta de apps para niños y jóvenes y, de una forma argumentada, exponer el valor de un determinado título en razón de unos criterios de análisis y evaluación objetivos, tanto de los aspectos formales como de los contenidos de una determinada aplicación, sea de ficción o de no ficción.

Es pues momento de aguzar la mirada y de poner en valor la oferta de obras digitales dirigidas a niños y jóvenes, de analizar las mimbres con las que están hechas y de destacar aquellos materiales que aportan calidad al conjunto, que se arriesgan a explorar nuevos caminos y que ofrecen al lector una experiencia de lectura más completa y rica a la par que rigurosa en su concepción y trazado. 
Ante este reto surgen plataformas que, como Apptk.es, buscan responder a las necesidades planteadas y pretenden convertirse en herramientas de referencia para la selección de aplicaciones de lectura infantil desde las que asentar criterios y parámetros de selección en torno a los que articular el análisis y la evaluación de aplicaciones de lectura. En el caso de la referida plataforma, se desarrolla paralelamente una tarea de sensibilización y formación en torno a la lectura digital que complementa su labor de orientación y recomendación (Figura 3).

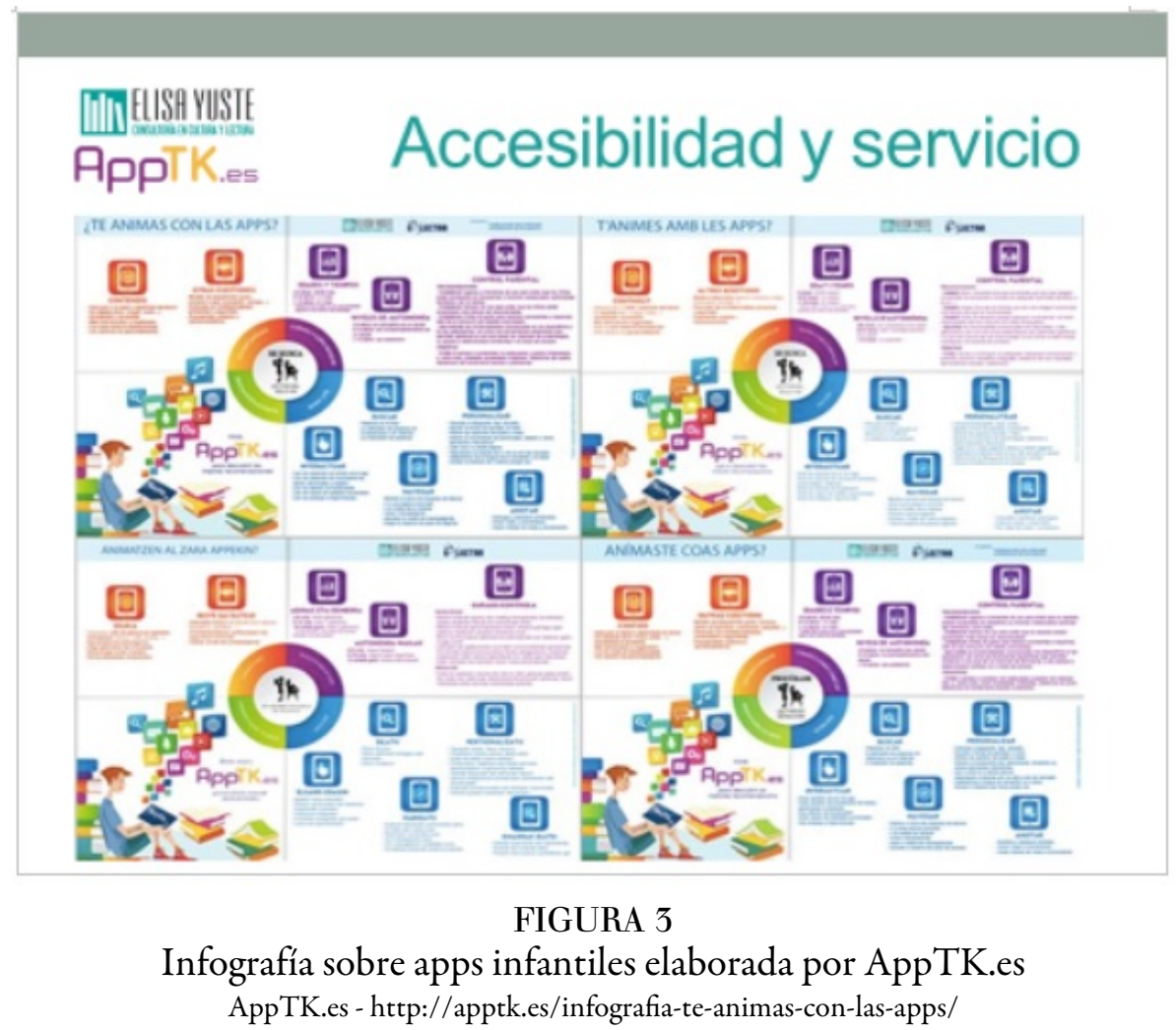

De esta manera se ayuda a los docentes de los diferentes niveles educativos, bibliotecarios infantiles y juveniles, madres y padres, desarrolladores de aplicaciones, editoriales y otros agentes implicados, a navegar en estos procelosos mares y a sacarle un mayor partido a la lectura digital y a las apps dirigidas al público infantil y juvenil en particular.

\section{Caracterización de las diferentes tipologías de RECOMENDADORES}

Como punto central de esta reflexión nos marcamos conocer más a fondo los sistemas de recomendación de aplicaciones infantiles, los entornos en los que se desarrollan y ofrecer un panorama general sobre estas herramientas.

En primer lugar, nos planteamos realizar una búsqueda de antecedentes sobre el tema y conocer el estado de los estudios.La búsqueda de material bibliográfico se realizó a través de tres bases de datos, dos dedicadas al mundo de la información y la documentación (LISA y LISTA) y otra al de la educación (ERIC). Se recurrió también al repositorio Dialnet, a Google Scholar y a un motor de búsqueda genérico (Google). Los descriptores utilizados fueron recomendaciones, aplicaciones y niños. Los trabajos se buscaron tanto en español como en inglés a partir de 2010. Aunque los resultados obtenidos fueron reducidos, se han encontrado algunos artículos y sitios web útiles. En el primer grupo se incluyen el artículo sobre criterios de evaluación de las aplicaciones de lectura infantiles de García-Rodríguez y Gómez-Díaz (2015); los trabajos de Chen, Xu, Zhou y Zhu (2013) que analizan los criterios de evaluación de las tiendas de Android y iOS; y el de Kucirkova, 
Littleton y Cremin (2016) sobre lectura por ocio infantil y libros digitales. En el segundo, las organizaciones sin ánimo de lucro Common Sense Media y Literacy Apps, con apartados sobre la importancia de seleccionar aplicaciones aptas a las necesidades de los niños y consejos para los mediadores sobre cómo utilizarlas.

Como se puede deducir de los resultados de las búsquedas, el tema no ha recibido particular atención a nivel académico. Sin embargo, encuentra mayor importancia y difusión en blogs y páginas dedicadas a los mediadores.

En segundo lugar, escogimos un grupo de recomendadores para analizarlos y estudiar sus características. Para ello, se partió del trabajo de García-Rodríguez y Gómez-Díaz (2016) en el que se recogen 19 plataformas de recomendaciones y 9 blogs dedicados a la lectura infantil en los entornos digitales. Tras una primera evaluación general, seleccionamos 19 recomendadores: Literacy Apps, Best Apps for Kids, La Souris Grise, Le Petite Bibliothèque Ronde- BibApps, AppTK, Boolino, Apps Playground, Appyautism, ID BOOX, Moms with apps, Super Julie- Le top de applis pour enfants, Know What's Inside, Los Cuentos de Bastian, Smart Apps for Kids, Déclic Kids- Digital Media for Kids, Digital Storytime, Generación APPS, Frikids y The App Date.

En tercer lugar, procedimos a seleccionar las variables de análisis y a crear una plantilla en la que recoger los resultados de las observaciones realizadas. La base de esta labor han sido diferentes artículos, como Codina (2000), Ayuso García y Martínez Navarro (2006a) y Galina Russell (2016), para lo relacionado con la autoridad, actualización de contenidos, ergonomía y adecuación al destinatario, y los de Ayuso García y Martínez Navarro (2006b) y García-Romero y Faba-Pérez (2015) sobre la evaluación de bibliotecas digitales, que se utilizaron para los aspectos relacionados con la navegabilidad de los sitios web. En todos los casos, se han adaptado los contenidos de cada artículo a los criterios necesarios para evaluar los recomendadores.

Se han recopilado las informaciones base, como el nombre, el URL, el idioma, la dependencia, el tipo de sitio web y las modalidades de financiación adoptadas. Estos parámetros no han sido considerados a la hora de recoger datos, sino que han servido para identificar y distinguir las diferentes tipologías de recomendadores con las que se estaba trabajando. Luego, se han analizado las características del sitio web a través de parámetros que evalúan la credibilidad, la posibilidad de navegación, puesta al día de los contenidos, estructura y coherencia de los contenidos con respecto a los destinatarios seleccionados, la interacción de la plataforma con los usuarios o con otros agentes como desarrolladores, ilustradores, etc., y su presencia en las redes sociales.

\section{RECOGIDA DE DATOS Y OBSERVACIONES SOBRE LOS RESULTADOS}

Como se explicó anteriormente, se analizaron 19 recomendadores de aplicaciones infantiles cuyos datos se recogieron entre los meses de enero y febrero de 2017, según una plantilla preestablecida. A cada uno de los indicadores seleccionados podía corresponder una evaluación positiva o negativa, que indicaban respectivamente su efectivo o no cumplimiento. A cada respuesta positiva se hizo corresponder 1 punto, por las respuestas negativas no se asignó ni restó ningún punto.

Si se analizan los puntajes totales obtenidos por cada recomendador, se puede observar que el que ha obtenido el porcentaje más elevado (79\%) ha sido Literacy Apps, sitio web creado por el National Literacy Trustcon el apoyo de diferentes universidades y partners inglesas. Se ha destacado particularmente por los parámetros “autoridad”, “actualización de contenidos", "ergonomía”, "adecuación al destinatario", "selección de contenidos", "evaluación de aplicaciones” y por la calidad de los recursos ofrecidos.

A continuación, se encuentran las dos plataformas que han obtenido un porcentaje entre 75-77\%: BestAppforKids y La Souris Grise, dos sitios diferentes entre ellos por tipología y por destinatarios. El primero, de origen norteamericano y mantenido por una madre, es un blog quese encarga de evaluar aplicaciones y guiar a los padres en la selección de contenidos, exclusivamente para iOS. La segunda es una página web francesa dedicada a diferentes aspectos relacionados con el mundo de las aplicaciones infantiles: evaluaciones y reseñas, noticias sobre tecnología y mundo infantil, estudios sobre lectura digital. 
En la siguiente banda (73-75\%), se colocan tres recomendadores: Le Petite Bibliothèque Ronde- BibApps, un catálogo de aplicaciones en línea para bibliotecas realizado por expertos de diferentes sectores como psicólogos, editores digitales, escritores, bibliotecarios, etc.; AppTK, página por expertos y profesores de la Universidad de Salamanca que reseñan aplicaciones infantiles, con particular atención a las apps literarias, y Boolino, el Bookadvisor para niños y jóvenes lectores, que une el "tradicional” sector editorial y el mundo de las appsy de los ebooks.

Cinco recomendadores han obtenido un 70\%: Apps Playground, Appyautism, ID BOOX, Moms with apps y Super Julie- Le top de applis pour enfants. Es interesante observar que entre ellos se encuentran plataformas muy diferentes por los parámetros cumplidos, por la calidad de los contenidos que ofrecen, organización, objetivos, etc.

Entre los recomendadores que han conseguido entre un 68\% y 66\%, se encuentran los blogs de padres Know What's Inside, Generación APPS, Smart Apps for Kids, Déclic Kids- Digital Media for Kids, Digital Storytime y la tienda online Los Cuentos de Bastian .

Solo un recomendador ha quedado por debajo del 50\%: The App Date, una web genérica de aplicaciones con una sección dedicada a las infantiles, que carece de indicadores importantes, tales como los de autoría u objetivos, y no cumple con la mayoría de los parámetros de navegabilidad.

El acceso a contenidos digitales de calidad y fiables es uno de los principales desafíos para los mediadores: los recomendadores deben proporcionar información valiosa a sus usuarios. Ahora bien, es importante consignar algunos indicadores que pueden ayudar a establecer la calidad y la credibilidad de dicha página.

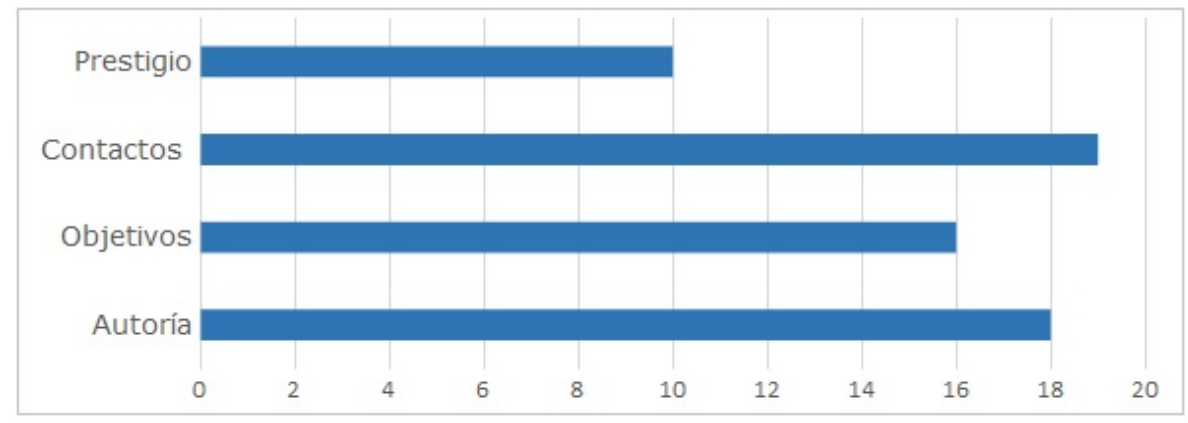

GRÁFICO 1

Comportamiento del parámetro "autoridad"

Elaboración propia

El parámetro "autoridad" recoge datos sobre la responsabilidad intelectual y la identificación de los responsables del sitio. Su objetivo es conocer con precisión quién se ocupa de los contenidos que el sitio propone y cuáles son sus finalidades.

En lo relativo a la autoría, el $94 \%$ de los recomendadores indican claramente quiénes son los responsables del sitio, salvo The AppDate. En cuanto a la definición de objetivos de la web, como se puede observar en el Gráfico 1, la mayoría de los recomendadores (84\%) dispone de un apartado específico. Todos los recomendadores disponen de alguna modalidad para que los usuarios puedan ponerse en contacto con los responsables del sitio. La relación con los usuarios es fundamental en este tipo de portales para recibir un feedback sobre el trabajo que se está llevando adelante, atender sugerencias de mejora, conocer qué tipo de usuarios utilizan la web y con qué fin, saber si las recomendaciones son útiles, etc. Algo más de la mitad de los recomendadores (53\%) ha recibido premios, menciones o reconocimientos por parte de otros recomendadores, blogs o instituciones: por ejemplo, el blog Digital Storytime ha creado una red de páginas web dedicadas a las reseñas de apps y tecnología; LiteracyApps es una página web creada y soportada por el National Literacy Trust para fomentar la alfabetización y la lectura en Reino Unido.

El apartado "navegabilidad"es particularmente importante dada la función que tiene el buscador dentro de los recomendadores como instrumento que permite encontrar, seleccionar e individualzar las aplicaciones 
más adecuadas a las necesidades de los usuarios. Solo el 25\% de los recomendadores cumple con el indicador "mapa de navegación", es decir, ofrecen un apartado en el que se describen con precisión los contenidos disponibles. Aunque puede resultar a priori un dato llamativo, hay que tener en cuenta que su falta no puede considerarse un aspecto negativo si la página web es particularmente ordenada y fácil de usar, una característica que cumplen la gran mayoría de los recomendadores analizados. Entre los sistemas de búsqueda, es evidente la predilección por la búsqueda libre (79\%), aunque se incorporan algunos filtros para afinar las búsquedas, frente al 11\% que cuentan además con búsqueda avanzada, concretamente Appyautism y Bibapps. En lo que se refiere a los filtros para realizar las búsquedas (Gráfico 2), el de la edad es el más común; esto es lógico si tenemos en cuenta que la mayoría de los recomendadores están dirigidos a adultos, para quienes este es un criterio muy utilizado a la hora de realizar una compra.

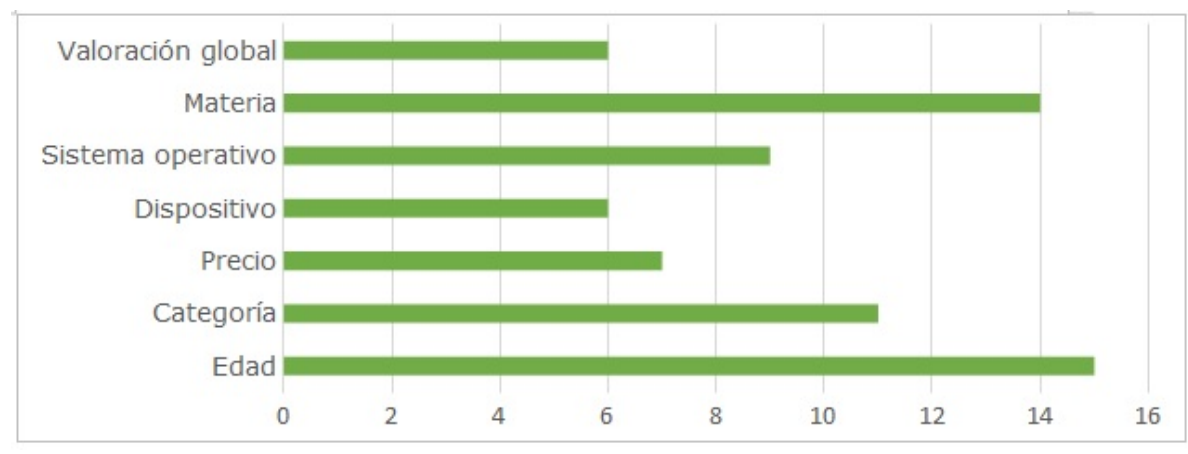

GRÁFICO 2

Filtros que se ofrecen para realizar las búsquedas

Elaboración propia

Por este motivo no sólo es un filtro importante sino también un criterio de evaluación que se encuentra en la mayoría de las reseñas, aunque es importante señalar la falta de uniformidad en cuanto a la delimitación de los tramos de edad. El que un recomendador permita limitar la búsqueda por este concepto, ayudará a los mediadores a seleccionar y comprar apps para sus hijos; si esta es una dinámica habitual en la literatura impresa, ¿por qué no se hace lo mismo para la edición digital? (García Rodríguez, 2013). Otro filtro presente en más del $70 \%$ de los sitios es el de materia: se trata de un opción de búsqueda que siempre debería estar, posiblemente acompañada por el browsing de materias específicas (García Rodríguez, 2013), ya que la aplicación de tags y la asignación de materias son aspectos que facilitan la búsqueda y el descubrimiento de nuevos productos. Solo la tienda online Los Cuentos de Bastian ${ }^{\bullet}$ dispone de buscadores y filtros lo suficientemente intuitivos para que los pueda utilizar un niño. El resto de los buscadores no parece estar pensado para niños, un dato particularmente llamativo pero no nuevo que ya se encuentra en la literatura infantil y juvenil en papel: son los adultos quienes seleccionan y compran los contenidos, por lo tanto las páginas web se adaptan más a sus necesidades que a las del propio público infantil. En menos de la mitad de los recomendadores es posible filtrar las búsquedas según los dispositivos y los sistemas operativos. El primer indicador puede considerarse poco útil, dado que actualmente la mayoría de las aplicaciones están disponibles para diferentes dispositivos, pero el segundo es un indicador importante, aunque poco común, dado que permite seleccionar entre los resultados los sistemas operativos para los que las aplicaciones están disponibles. Esta falta a la hora de afinar una búsqueda se compensa parcialmente con el hecho de que en el $89 \%$ de las reseñas haya un apartado dedicado a ello.

El mercado de las aplicaciones infantiles ofrece continuamente nuevos contenidos: padres, maestros y cualquiera que esté involucrado en la educación de los niños necesita puntos de referencia para poder enfrentarse a estos cambios. Entre los recomendadores seleccionados, sólo uno de los analizados no se ha actualizado en la fecha de consulta, el resto se actualizan constantemente aunque con diferente regularidad, si bien es necesario matizar que la mayoría de los recomendadores no incluye información directa sobre el tema. 
En el parámetro "ergonomía" se incluyen todos los elementos del sitio que permiten una navegación sencilla y favorecen la lectura de los contenidos. Estándares elevados en este parámetro facilitan la navegación y la lectura de los contenidos gracias a una buena relación entre figura-fondo, a un adecuado tamaño de tipografía, etc. La mayoría de los recomendadores (89\%) se caracteriza por una cierta facilidad de navegación y permiten una lectura sencilla de los contenidos. Todos cumplen con los indicadores "claridad y contraste" y "legibilidad", y es posible reconocer un particular cuidado en su esquema de distribución de los elementos del diseño de la página, un buen equilibrio entre texto-fondo, ilustraciones-texto, ilustraciones-fondo y una tipografía nítida. Es significativa también la diferencia entre la ergonomía de los sitios institucionales y los personales, seguramente debido a la disponibilidad de mayores recursos para contratar servicios específicos de desarrollos web profesionales con conocimientos sobre el tema por parte de los primeros, frente al uso de software libre y mantenimiento personal en el segundo caso.

El apartado dedicado a la adecuación al destinatario se centra en la compatibilidad y la pertinencia entre los intereses de los destinatarios y los contenidos disponibles: es importante que sean aptos a las necesidades de los usuarios a los que se dirigen, ya sean niños o mediadores. En todos los indicadores es posible determinar un destinatario preciso: la mayoría cuenta con una sección o un apartado donde aclara hacia quién están dirigidas las reseñas (Gráfico 3).

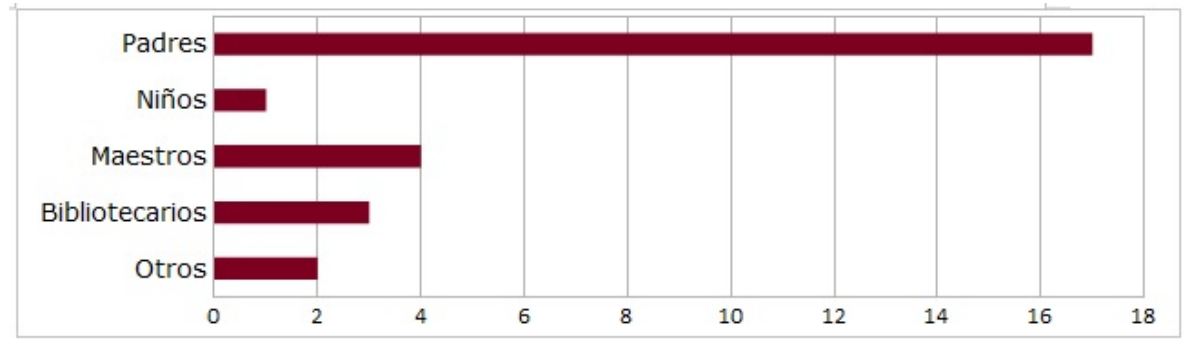

GRÁFICO 3

Destinatarios de las reseñas

Elaboración propia

Como se observa en este gráfico, los principales destinatarios de las recomendaciones son padres, seguidos por maestros y bibliotecariosLosCuentos de Bastian ${ }^{\circledR}$ es el único recomendador adaptado realmente al público infantil en "navegabilidad" y en el tipo de recomendaciones que ofrece. Dentro del apartado "Otros", podemos encontrar algunos recomendadores especializados en personas con TEA (Trastorno del Espectro Autista) como Appyautism, y a los profesionales del mundo editorial como La Souris Grise. Podemos afirmar por tanto que los recursos analizados se dirigen a un público variado, básicamente adulto, pero son pocos los que piensan en los usuarios finales, los niños.

En lo que se refiere a la información sobre descuentos, ofertas y demás, la cifra obtenida (37\%) confirma que son pocas las plataformas que recogen esta información a través de la relación con las tiendas o los desarrolladores. Una de las formas más populares de obtención de recursos (incluida por el 63\% de los evaluadores) consiste en la posibilidad de ofrecer servicios adicionales a los desarrolladores. Entre los servicios ofrecidos se incluyen la opción de evaluación para la mejora en los desarrollos, la promoción de las aplicaciones entre los lectores y la oferta de formación específica para padres, maestros y bibliotecarios.

Las recomendaciones por parte de los usuarios son fundamentales para la difusión de los contenidos infantiles. Por este motivo, es importante que los recomendadores otorguen la posibilidad de comentar cada reseña o de compartirla en diferentes redes sociales.

Según los resultados obtenidos, más de la mitad de los recomendadores (un 68\%) facilitan la primera opción. Sin embargo, es la importancia de las redes sociales como medio de descubrimiento y recomendación de lecturas la que destaca especialmente, con un $84 \%$ de resultados positivos obtenidos (Gráfico 4). Esto se debe principalmente a que plataformas como Facebook, Twitter, Youtube o Instagram (entre otras) facilitan 
la posibilidad de compartir comentarios y reseñas propias, permitiendo así mismo la interacción con las propuestas de otros usuarios.

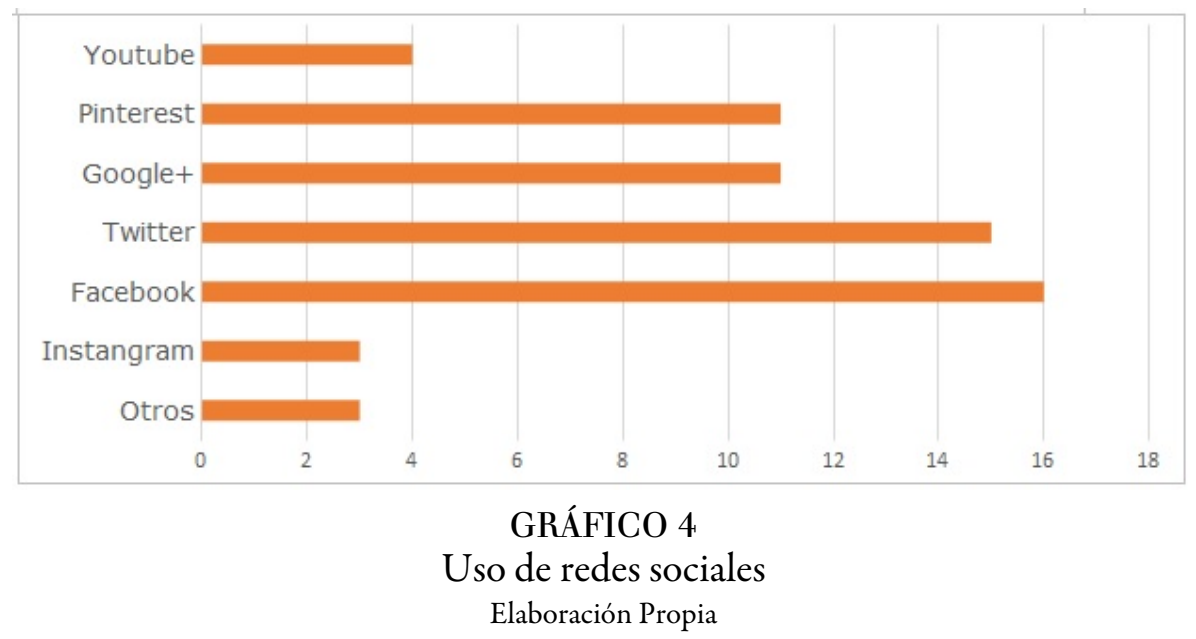

Una muestra efectiva de esta influencia puede verse en la cantidad de blogs, canales de Youtube y cuentas de Instagram dedicadas a la creación de reseñas y videoreseñas de literatura infantil y juvenil donde, en muchos casos, los usuarios dedican sus esfuerzos a guiar a los adultos en la selección de los mejores contenidos para los más pequeños.

De entre todas, Facebook es la red social que ostenta el título de "predilecta" con un 80\% de recomendadores y más de 25 millones de usuarios activos al mes en España (Hootsuite, We Are Social, 2017). Una posible relación entre la superioridad de Facebook y la escasa popularidad de otras redes, a pesar de que son bastante utilizadas por los consumidores, está en la propia estructura de la red social. Facebook ofrece una opción de comentarios ilimitados, sin necesidad de contar caracteres, con la posibilidad de controlar quién interacciona con el contenido, opción para grupos, posibilidad de incluir contenido multimedia de distinto tipo, etc.

\section{Criterios de eVAluación de las aplicaciones en Sí Mismas Y EN Relación con la ADECUACIÓN A LAS EDADES Y COMPETENCIAS DE LOS DESTINATARIOS}

En este contexto de herramientas de prescripción de apps queremos también ofrecer una relación de los criterios que pueden guiar el análisis de apps de lectura y libros app y la selección conforme a la edad y las competencias de los destinatarios. Estos criterios son (y si no lo son, deberían serlo) la base de trabajo de los servicios de recomendación de apps.

Cuando nos enfrentamos a una app de lectura o a un libro app, nuestra primera recomendación es echarle un primer vistazo al contenido para ir tomándole el pulso a la propuesta. $\mathrm{Y}$, una vez que hemos realizado esa primera aproximación, volver a ella e ir revisando uno a uno diferentes aspectos que enunciamos a continuación.

Veremos que muchos de los criterios que se utilizan en el análisis y selección de apps de lectura y libros app son los que tenemos presentes cuando abordamos materiales de lectura en formato impreso. Otros, en cambio, son aspectos más propios sino específicos de este tipo de propuestas de lectura en soporte digital.

La primera apreciación es fácil que esté relacionada con el contenido en términos generales: el tema, su originalidad, su interés y su adecuación en relación con el lector-usuario para el que ha sido concebida la app, su planteamiento y su enfoque también teniendo presente, de nuevo, al lector-usuario.

También se habrán de analizar sus elementos constitutivos: texto, imagen (estática y en movimiento), audio (narración, efectos de sonido, banda sonora), elementos interactivos, propuestas de gamificación... Y 
valoraremos su calidad de forma independiente pero también la relación que establecen entre ellos en el entorno de la app.

En el caso de las apps de lectura, además, puede tenerse en cuenta la cantidad y la variedad de temas, géneros y tipologías de contenido, así como el ritmo de incorporación de nuevos títulos. Por otra parte, en el caso de que el contenido sea de no ficción se habrá de medir la claridad y la objetividad en el tratamiento del tema, si está actualizado.

Otro aspecto en el que poner la mirada es la organización de los contenidos y, consecuentemente, su visualización dentro de la aplicación. Es importante que se facilite al lector-usuario la navegación por el entorno de la app. Por ello puede ser de interés que favorezca la accesibilidad al contenido a través de una o varias herramientas de búsqueda, con o sin conexión a Internet.

En el caso de las apps de lectura, además, es interesante que incorporen opciones de personalización para la organización del contenido, por ejemplo, en carpetas, para que los lectores-usuario autónomos puedan categorizar el contenido en función de sus gustos, intereses y necesidades. Incluso, que permita eliminar y restaurar contenidos en función de los intereses del lector-usuario.

Siguiendo con las opciones de personalización, un aspecto en el que tienen un amplio espectro de posibilidades las propuestas digitales, podemos considerar desde las posibilidades de personalizar los aspectos formales del texto, del audio, del vídeo y la animación.

En el caso del texto, el tipo y el tamaño de letra, los ajustes de brillo y fondo de pantalla, la lectura a una o a doble página; incluso, la disponibilidad de lectura en varios idiomas o variantes idiomáticas. En el caso del audio, la elección de narrador, la grabación personalizada de la historia, los efectos de audio o banda sonora. Y en el caso del vídeo y la animación: su activación automática o no.

Las funcionalidades relativas a la búsqueda de palabras o la incorporación de anotaciones también pueden ser de interés con lectores-usuario más autónomos. En estas edades más avanzadas también se pueden valorar las posibilidades de interacción con los contenidos que permitan a los lectores compartir lecturas, comentarlas, etc. En cualquier caso, se habrá de comprobar la adecuación y el nivel de dificultad de este tipo de acciones.

Otro aspecto, por lo general, diferencial de los contenidos digitales (aunque no exclusivo) en el que poner la mirada es la interactividad. Por ello es conveniente comprobar si el contenido de la app es interactivo y los niveles de interactividad que brinda al lector-usuario: si es residual o si permite al lector actuar y participar como un personaje más de la historia y tomar decisiones en el proceso de lectura. Algo que, como el resto de aspectos, tendremos que valorar en función del usuario y las habilidades que se le imaginen.

También podemos echar un vistazo a los créditos, que en este tipo de contenidos se multiplican exponencialmente (autor, ilustrador, diseñador, desarrollador, compositor...). En algunos casos puede ser una información significativa, especialmente cuando el mediador cuente con cierta trayectoria en el análisis y selección de apps. Es significativo que se incluya, en cualquier caso, aunque a la persona que esté haciendo el análisis no le diga mucho de primeras.

También puede dar pistas la fecha de creación y las de las diferentes versiones de la herramienta, si las hubiera. Por ejemplo, una app que lleve tiempo en el mercado puede ser sinónimo de éxito, al igual que una app que lleve a sus espaldas múltiples actualizaciones que, además, pueden estar mostrando la preocupación de su desarrollador por optimizar su propuesta.

Una vez valorado el contenido, es clave abordar aspectos como la ergonomía, y tener en cuenta todos los aspectos que pueden favorecer la visualización del contenido de la app de una forma sencilla y clara: el tamaño y el tipo de letra, el contraste de la letra con el fondo, el uso del color, la colocación de los diferentes elementos e iconos dentro de la página, la claridad de las imágenes, la utilización de botones fáciles de identificar, etc.

Junto a la ergonomía también habremos de valorar la usabilidad y determinar el grado en el que los usuarios interactúan con la aplicación de manera eficiente e intuitiva. Una característica que está relacionada con la facilidad de uso, con la rapidez con la que se puede aprender a utilizar y con el grado en el que su diseño facilita 
su manejo. Este parámetro está directamente relacionado con la accesibilidad y la ergonomía, pero también con la velocidad de apertura de la app, la navegación.

Muy importante en relación con el público que nos atiende son los aspectos relativos a la seguridad, la confidencialidad, la privacidad. Así habremos de valorar que incluya funcionalidades de control parental o bloqueo de compras dentro de la app, así como que las claves de acceso sean acordes a la edad del usuario.

Además, las aplicaciones concebidas para menores deben cumplir las diferentes leyes vigentes en cada país de origen sobre protección de datos, garantizar la confidencialidad de sus usuarios y proteger la privacidad de la información ofrecida por ellos, datos que deben aparecer de una forma clara y explícita en el producto.

Es necesario comprobar qué datos se solicitan y cuáles se recogen de forma automática (dirección IP, identificación del dispositivo...), y el uso de esos datos. Las aplicaciones concebidas para menores no deben solicitar ningún tipo de información personal de los menores para activar su funcionamiento, mucho menos sin el consentimiento de un adulto responsable.

Otros aspectos a tener en cuenta de cara a la selección son la disponibilidad y las cuestiones relativas a la adquisición. En relación con la primera habremos de valorar el nivel de acceso a la app y a sus contenidos: su disponibilidad para diferentes sistemas operativos. También se puede valorar positivamente que esté disponible en diferentes tiendas, idiomas (de uso de la aplicación), que sea fácilmente localizable (por título, icono). Todo ello amplía el espectro de lectores potenciales.

En cuanto a la adquisición, más allá del precio, hay que analizar la forma de adquisición del contenido y determinar si es adecuada: venta unitaria, bundle, suscripción (mensual, anual), gratis (con publicidad, sin publicidad, versión lite). Son especialmente valorables las que ofrecen diferentes opciones y permiten elegir al usuario en función de sus intereses y necesidades.

También se pueden tener presentes, aunque con todas las reservas puesto que no constituyen indicadores de calidad, la popularidad de la app (elevado número de descargas, buena puntuación en base a un buen número de valoraciones, comentarios positivos de los usuarios...) y el reconocimiento (premios, sellos de calidad).

Una vez analizados estos parámetros, se puede hacer una suma y ver cuál sería la valoración de la aplicación en base a ella. No se puede rechazar una app porque flaquee en algún aspecto. Además, no siempre podremos utilizar los mismos criterios. E insistimos en que, por encima de todo, se ha de tener presente la edad de los lectores-usuario para los que ha sido creada la app, sus gustos, sus intereses y sus habilidades.

Si tenemos en cuenta el elevado número de aplicaciones disponibles en el mercado y su crecimiento exponencial, el análisis y selección de propuestas de calidad se hacen cada vez más necesarios para motivar a los niños y jóvenes en la lectura digital, fomentar el hábito lector en los nuevos soportes y desarrollar sus habilidades como lectores del Siglo XXI.

\section{CONCLUSIONES}

De todo lo antedicho se pueden extraer una serie de conclusiones que reafirman el interés y necesidad de crear plataformas de evaluación y recomendación de aplicaciones y otros materiales de lectura digitales, de ficción y no ficción, dirigidas a niños y jóvenes. Destacamos a continuación algunos de los aspectos abordados que resultan más significativos respecto al tema tratado:

- La oferta de aplicaciones, y otras obras digitales dirigidas al público infantil, experimenta una constante expansión y un crecimiento cualitativo y cuantitativo que requiere de mayor atención por parte de los espacios de recomendación y crítica.

- La actual oferta de materiales digitales de lectura para niños y jóvenes es poco visible y difícil de identificar, por lo que se hace necesario poner a disposición de padres, docentes, bibliotecarios y otros 
agentes de promoción de la lectura, herramientas de orientación y recomendación que faciliten la integración de las obras digitales en la familia, la escuela y la biblioteca.

- El análisis del estado de la cuestión de los recomendadores de apps para niños y jóvenes, si bien arroja la existencia de plataformas de interés, pone de manifiesto que se necesita reforzar su presencia en el ámbito lingüístico del español, así como en el de las otras lenguas ibéricas. Identificar y evaluar los materiales producidos en territorios de estos ámbitos lingüísticos, o que incluyen opciones de lectura en estas lenguas, se presenta como una necesidad y una oportunidad para complementar la acción que otros recomendadores ya realizan en otras áreas geográficas y lingüísticas.

- Las plataformas de recomendación deben regirse por una serie de claves de actuación que aseguren su transparencia, la aplicación de criterios definidos a la hora de valorar y recomendar lecturas, así como la construcción de interfaces accesibles, de fácil uso y con opciones de búsqueda adecuadas.

- En cuanto a los contenidos ofrecidos por estas plataformas, se destaca la necesidad de que ofrezcan datos de identificación y acceso a los materiales seleccionados; que los reseñen no sólo de forma descriptiva sino de forma crítica, y que aporten a la comunidad de usuarios potenciales a los que se dirigen elementos de formación en el uso de las obras digitales que favorezcan una mayor y mejor implantación de estas obras en los distintos entornos en los que se mueven los niños y jóvenes.

- En relación con los destinatarios de estas plataformas, queda patente que se dirigen, salvo alguna leve excepción, al público adulto, como de forma recurrente viene ocurriendo en los espacios de orientación de recomendación de literatura infantil y juvenil, independientemente de que sean estos impresos o digitales y de que sus contenidos sean obras impresas o de lectura en pantalla.

\section{ReFERENCIAS}

Ayuso García, D., y Martínez Navarro, V. (2006a). Evaluación de calidad de fuentes y recursos digitales: guía de buenas prácticas. Anales de Documentación, 9, 17-42. Recuperado de http://revistas.um.es/analesdoc/article/view/184 $1 / 1841$

Ayuso García, D., y Martínez Navarro, V. (2006b). Metodología de evaluación de recursos en bibliotecas digitales. Parámetros e indicadores de calidad. Ciencias de la Información, 37(1), 25-44. Recuperado de http://cinfo.idic t.cu/index.php/cinfo/article/viewFile/95/94

Chen, Y., Xu, H., Zhou, Y., y Zhu, S. (2013). Is This App Safe for Children? A Comparison Study of Maturity Ratings on Android and iOS Applications. En Proceedings of the 22nd international conference on World Wide Web, 201-211. Recuperado de https://faculty.ist.psu.edu/xu/papers/www2013.pdf

Codina, L. (2000). Evaluación de recursos digitales en línea: conceptos, indicadores y métodos. Revista española de Documentación Cientifica, 23(1). http://dx.doi.org/10.3989/redc.2000.v23.i1.315

Galina Russell, I. (2016). La evaluación de los recursos digitales para las Humanidades. Signa: revista de la Asociación Española de Semiótica, 25, 121-136. Recuperado de http://www.cervantesvirtual.com/obra/evaluacion-recurso s/

García-Rodríguez, A. (2013). El papel de las plataformas de distribución y venta en la promoción del libro electrónico infantil y juvenil. Anales de Documentación, 16(1). http://dx.doi.org/10.6018/analesdoc.16.1.166601

García-Rodríguez, A., y Gómez-Díaz, R. (2015). Las demasiadas aplicaciones: parámetros e indicadores para seleccionar las topapp de lectura para niños. Anales de Documentación, 18(2). http://dx.doi.org/10.6018/anale sdoc.18.2.227071

García-Rodríguez, A., y Gómez-Díaz, R. (2016). Lectura digital infantil: dispositivos, aplicaciones y contenidos. Barcelona: UOC.

García- Rodríguez, A., Gómez-Díaz, R. y Cordón-García, J. A. (2013): De Alicia en el país de las maravillas a las maravillas de Alicia. Ibersid. 7, 21-32. Recuperado de http://www.ibersid.eu/ojs/index.php/ibersid/article/vie wFile/4092/3743 
García-Romero, J. E. y Faba-Pérez, C. (2015). Desarrollo e implementación de un modelo de características o indicadores de calidad para evaluar los blogs de bibliotecas escolares de centros de educación infantil y primaria. Revista española de Documentación Cientifica, 38(1). http://dx.doi.org/10.3989/redc.2015.1.1169

Hootsuite, We Are Social. (2017). Digital in 2017: Southern Europe. We Are Social. Recuperado de http://www.slid eshare.net/wearesocialsg/digital-in-2017-southern-europe

Kucirkova, N., Littleton, K., y Cremin, T. (2016). Young children's reading for pleasure with digital books: six key facets of engagement. Cambridge Journal of Education, 47(1), 67-84. https://doi.org/10.1080/0305764X.201 5.1118441

Observatorio de la Lectura y el Libro (2017). Los libros infantiles y juveniles en España. Ministerio de Educación, Cultura y Deporte. Recuperado de http://www.mecd.gob.es/dam/jcr:55a802b2-37b9-463e-8227-1f94a2b142 13/InformeLIJ-2017.pdf

Riaza, M. (2016). Infografía “Cómo encuentran los lectores su próxima lectura”. Dosdoce.com. Recuperado de http: //www.dosdoce.com/2016/05/09/infografia-encuentran-lectores-proxima-lectura/

We are social (2017). Digital in 2017: Global Overview. Report from We Are Social and Hootsuite. Recuperado de h ttp://wearesocial.com/blog/2017/01/digital-in-2017-global-overview

\section{Anexo}

\section{Recomendadores analizados}

- Literacy Apps http://literacyapps.literacytrust.org.uk/

- Best Apps for Kids https://www.bestappsforkids.com/

- La Souris Grise http://www.souris-grise.fr/

- Le Petite Bibliothèque Ronde- BibApps http://www.bibapps.com

- AppTK http://apptk.es/

- Boolino https://www.boolino.es/es/

- Apps Playground http://appsplayground.com

- Appyautism http://www.appyautism.com/

- ID BOOX https://www.idboox.com/

- Moms with apps http://blog.momswithapps.com/

- Super Julie- Le top de applis pour enfants http://www.super-julie.fr/

- Know What's Inside (redirect to https://actonline.org/privacy/)

- Los Cuentos de Bastian http://www.loscuentosdebastian.com/

- Smart Apps for Kids http://www.smartappsforkids.com/

- Déclic Kids- Digital Media for Kids http://digitalkidsmedia.com/

- Digital Storytime http://digital-storytime.com/

- Generación APPS http://generacionapps.com/

- Frikids http://www.frikids.com/

- The App Date http://www.theappdate.es/apps/categoria/android/ninos/ 